\title{
Solving quadratic assignment problem using water cycle optimization algorithm
}

\author{
Maryam Parhizgar, Farhad Mortezapour Shiri \\ Department of Computer Engineering, Sience and Research Branch, Islamic Azad University, Qazvin, Iran \\ Email address: \\ mail.parhizgar@Gmail.com (M. Parhizgar),farhad.mortezapour.sh@Gmail.com (F. M. Shiri)
}

\section{To cite this article:}

Maryam Parhizgar, Farhad Mortezapour Shiri. Solving Quadratic Assignment Problem Using Water Cycle Optimization Algorithm. International Journal of Intelligent Information Systems. Special Issue: Research and Practices in Information Systems and Technologies in Developing Countries. Vol. 3, No. 6-1, 2014, pp. 75-79. doi: 10.11648/j.ijiis.s.2014030601.24

\begin{abstract}
The Quadratic Assignment Problem (QAP) is one of combinatorial optimization problems which devote some facilities to some locations. The aim of this problem is assignment of each facility to a location which minimizes total cost. Because the QAP is NP-hard, so it couldn't be solved by exact methods. In recent years, meta-heuristic algorithms are used in solving NP-hard optimization problems increasingly. In this article Water Cycle Optimization Algorithms (WCO) is used to solve QAP. The implementation of proposed algorithms on standard test functions and also its result comparison with other meta-heuristics algorithms express algorithm`s desirable quality and its prominence to other meta-heuristics algorithms.
\end{abstract}

Keywords: Quadratic Assignment Problem, Combinatorial Optimization Problems, Water Cycle Optimization Algorithms, Meta-Heuristics Algorithms

\section{Introduction}

The Quadratic Assignment Problem (QAP) is one of combinatorial optimization problems that, in many firms have done various researches in order to devote some facilities to some locations. The most important thing in this process is assignment costs, which this type of costs become minimized. The computation of optimized solutions is really hard and difficult for most of optimization problems observed in many operational and practical fields. The solutions for optimization problems include two groups of exact and heuristics methods. The exact methods gain optimized solution and guarantee optimizing condition [1]. Heuristics methods produce high qualified solutions in sensible time, but didn't guarantee to find the thorough optimized solution [2]. The QAP is a problem with exponential complexity. Totally, problems with larger than 20 dimensions aren't solvable through exact methods otherwise from the view point of time they aren't economical. Therefore, meta-heuristics methods are used in these cases. Indeed meta-heuristics algorithms is one of heuristics optimization algorithms types which have outgo mechanisms from local optimization and are applicable in many different problems [3]. Various types of these algorithms are developed during recent decades. The meta-heuristics algorithms which used in solving QAP are Annealing algorithm [4], Tabu Search
[5, 6], Genetics algorithm [7], Ant Colony Optimization algorithm [8, 9], Harmony Search algorithm [10], Imperialist competitive algorithm [11], Artificial Bee Colony algorithm [12], and Cuckoo algorithm [13].

In presented design, the syntax of WCO meta-heuristics algorithms are used to solve QAP. Implementation of proposed algorithm on standard test functions and also its comparison with other meta-heuristics algorithms express desirable quality and its prominence to other meta-heuristics algorithms. The structure of the reminder of this paper is as follows: Section 2 is an introduction to QAP. In section 3 an introduction to WCO algorithm is given. In section 4, we describe the proposed algorithm based on WCO algorithm to solve the problem. Section 5 and 6 are dedicated to describe experimental results and paper conclusion, respectively.

\section{Definition of Quadratic Assignment Problem}

The QAP is one of combinatorial optimization problem which devote a category of facilities to a category of locations, along with cost that is function of distance and the 
flow among facilities in addition to relevant costs to facilities which located in specified location. The aim of this problem is assignment of each facility to each location in way that total cost becomes minimized. General model of QAP is presented below.

$$
\begin{array}{cc}
\min \sum_{i=1}^{n} \sum_{j=1}^{n} \sum_{k=1}^{n} \sum_{l=1}^{n} f_{i j} d_{k l} x_{i k} x_{j l} \\
\text { s.t. } \sum_{i=1}^{n} x_{i j}=1 & 1 \leq j \leq n \\
\sum_{j=1}^{n} x_{i j}=1 & 1 \leq i \leq n
\end{array}
$$

Where $f_{i j}$ is the flow between $\mathrm{I}$ and $\mathrm{j}$ facilities, and $d_{k l}$ is the distance among $\mathrm{k}$ and 1 locations [14]. If pay attention to activities assignment to locations, general model of QAP is like below.

$$
\min \sum_{i=1}^{n} \sum_{j=1}^{n} \sum_{k=1}^{n} \sum_{l=1}^{n} f_{i j} d_{k l} x_{i k} x_{j l}+\sum_{i} \sum_{k} C_{i k} x_{i k}
$$

Kopmans and Beckman introduced QAP for the first time in 1957 as a mathematic model which is relevant to economic activities [15]. This problem has many different applications from that time up to now. One of the main usages of QAP is in Placement theory, which $\mathrm{F}=$ (fij) is a flow matrix. The aim of this problem is finding an assignment of all facilities to all locations with minimized cost. Other usages of it is sorting constructions in hospitals, warehouses management and distribution strategies, minimizing wire's cables length in electronic circuits, assignment of some factories to some locations, wiring the circuit problem, controlling panels and typist keyboards designing.

\section{Water Cycle Optimization Algorithm}

WCO Algorithm [16] is a meta-heuristics algorithm which is inspired from water cycle in nature. In presented scheme, there have been attempt to introduce a new algorithm using water cycle in nature. The aim of this problem is benchmarking WCO like raining, the population of each generation has suitable diversity and scattering, and also by simulation of falling water drops motion process toward river, algorithm has a high convergence speed to gain thorough optimum. In addition to benchmark saturated pits during raining, escaping designed local optimum make algorithm to have suitable search in problem space and doesn't trap into local optimum. This algorithm includes a set of primary values for each parameters of problem. In each cycle of performing this algorithm, some values are selected based on a priority for each parameter of problem and make a smaller category, then by considering values competence, several solution will be generated by incorporating values of each parameter. After that each solution of problem located in its local optimum using local search algorithm. Each local optimum according to its propriety receives a capacity that explains the survival of that answer in consecutive performances and also the probability of being candidate to move other answers towards itself. In each cycle of performance, the answers which should be exited out of problem move towards answers with better competence and reach to local optimum that chose by themselves, if they didn't meet any more competence answer during path. Each cycle one of problem solutions with the most competence reminds constant and at the end of each cycle, values of each parameter of problem solution enter to set of primary values. After ending $\mathrm{n}$ cycle, the best solution of $\mathrm{nth}$ cycle is optimum solution of problem.

\section{Water Cycle Algorithm Steps to Solve QAP}

Water-cycle algorithm has 5steps which include:

\subsection{Initialization}

Such as many evolutionary algorithms, this algorithm also initializes with set of random primary values. In first performance of it, ocean initialized by random primary values. It assumed for discrete problem solution, the answer is a permutation of 1 to $n$ (problems with $n$ variables).

Ocean and initialization: it is a $n^{*} n$ matrix that its initial value will be (Ocean Size) $/ n$, that Ocean size expresses size of ocean. In an example with 5 variables, ocean is a $5 * 5$ matrix and initial values of all its elements will be $200 / 5=$ 40. this values will change in river's water entrance into ocean. In this matrix, columns present a variable (tiny drop) of problem. Rows show the probability of locating one variable in location of 1 to $n$.

\subsection{Evaporation Step Out of Ocean and Formation of Cloud Droplets}

In this step some droplets should be selected for evaporation or formation of drop. This means that according to figures in each column, a droplet is selected in a way that more figure for a droplet, has more probability to choose. A simple solution can be in this way that sum first column droplets values, then generate a random value between 1 and sum of droplets value. This random value placed in intervals of each droplet, the droplet will be selected. Finally with $n$ droplet, one drop will be formed.

\subsection{Local Search}

In local search a drop should immigrate to its better neighbor. Three types of neighborhood are defined for permutation problems.

\subsubsection{Replacing Two Quantities}

Two locations selected randomly and their quantities replace with each other.

Example: 15423 , elements 1 and 4 are selected and result will be 13425

\subsubsection{Inverting a Limited Area}

Two randomly quantities are generated and the limited area between them will be inverted.

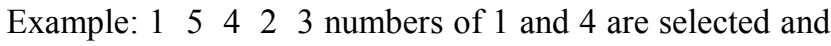


result will be 13245

Tow random quantities are generated and the first one converts to next element after second quantity.

Example: 15423 numbers of 1 and 4 are selected and result will be 1532

\subsection{Surface Runoff}

The way of falling water motion from the origin pit to destination pit is in this way that a permutation of numbers from 1 to $\mathrm{n}$ are generate randomly and based on that permutation, the droplet equal with the droplet on destination pit on that location, founded in origin pit and replaces with droplet in origin pit on that location.
Origin pit:
$\begin{array}{lllll}2 & 4 & 5 & 3 & 1\end{array}$
Destination pit:
$\begin{array}{lllll}4 & 3 & 2 & 1 & 5\end{array}$
Generated permutation: $\begin{array}{lllll} & 2 & 4 & 5 & 1\end{array}$

The origin pit after one step movement: $\begin{array}{llllll}1 & 3 & 2 & 4 & 5\end{array}$ the first number which generated by permutation is 3 , the third number of destination pit is 2 , that should be located in its main location in origin pit, it means that 2 replaces with 5 .

The origin pit after one step movement: $\begin{array}{lllll}5 & 3 & 2 & 4 & 1\end{array}$

The origin pit after one step movement: $\begin{array}{llllll}5 & 3 & 2 & 1 & 4\end{array}$

The origin pit after one step movement: $\begin{array}{lllll}4 & 3 & 2 & 1 & 5\end{array}$

Surface runoff to n-1 steps are needed.

\subsection{River Water Entry to Ocean}

The numbers inside ocean change like below in each step:

$$
\begin{gathered}
s_{i j}=s_{i j}-\left(\propto \times s_{-} i j\right) /(\text { Osean Sizae }) \\
s_{r_{i^{i}}}=s_{r_{i^{i}}}+\propto
\end{gathered}
$$

Where $\mathrm{S}$ is ocean itself, $\alpha$ is a coefficient which delivered after entry and $r$ is river.

\section{Experiments and Simulation Results}

This study addresses the general form of QAP and its purpose is to evaluate the WCO in solving QAP so that its applicability is confirmed and it can then be used in solving specific real cases in next studies. For this reason, those problems are chosen which are more famous and have been used for testing other algorithms. Therefore, the most credible reference of QAPs, QAPLIB [17], was used which is prepared by Peter Hann, Berkard, Chella, Randal, and Karisch who are mathematics professors that specialize in QAP. In QAPLIB, different QAPs of different sizes are defined and solved by scientists such as Berkard, Al-Shaafi, Steinburg, etc. using exact, heuristic, and meta-heuristic methods.

The results of experiments and simulations are presented in Tables 1, 2 and 3 and graphically in Figures 1 and 2. First, we determine the results for a small population. Then, the resulting error percent is calculated and compared to the genetic and honeybee and water cycle improved algorithms.

\begin{tabular}{|c|c|c|c|c|c|c|c|}
\hline $\begin{array}{l}\text { Problem } \\
\text { Name } \\
\end{array}$ & Problem Size & $\begin{array}{l}\text { Best Solution } \\
\text { Found So Far } \\
\end{array}$ & $\begin{array}{l}\text { Solution } \\
\text { Method } \\
\end{array}$ & $\begin{array}{l}\text { Errors of Previous } \\
\text { Methods }\end{array}$ & HBMO Error & $\begin{array}{l}\text { COA } \\
\text { Error } \\
\end{array}$ & $\begin{array}{l}\text { WCO } \\
\text { Error }\end{array}$ \\
\hline Lipa30a & 30 & 13178 & Exact & & 3.74 & 2.01 & 1.31 \\
\hline Lipa90a & 90 & 360630 & Exact & - & 1.67 & 0.39 & 0.28 \\
\hline Sko49 & 49 & 23386 & RO-TS & 5.91 & 16.11 & 3.56 & 0.10 \\
\hline Sko56 & 56 & 34458 & RO-TS & 5.37 & 18.49 & 4.39 & 1.04 \\
\hline Sko64 & 64 & 48498 & RO-TS & 5.7 & 16.91 & 4.47 & 1.41 \\
\hline Sko72 & 72 & 66256 & RO-TS & 5.38 & 14.34 & 4.85 & 1.34 \\
\hline Sko81 & 81 & 90998 & GA & $5 / 41$ & 13.57 & 4.332 & 1.38 \\
\hline Sko100a & 100 & 152002 & GA & $6 / 41$ & 12.74 & 12.95 & 1.48 \\
\hline
\end{tabular}

Table 1. Results of running the program with 200 repeats for the problems chosen from qaplib. errors are in percent.

Table 2. Results of running the program with 500 repeats for the problems chosen from qaplib. errors are in percent.

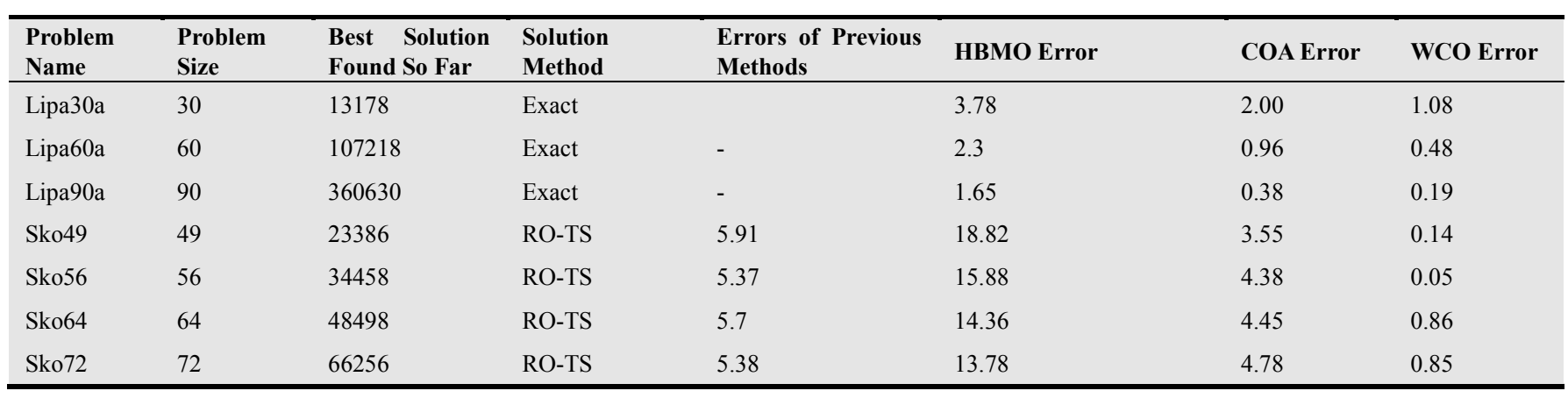


Table 3. Results of running the program for the problems chosen from qaplib with large population.

\begin{tabular}{lllllll}
\hline $\begin{array}{l}\text { Problem } \\
\text { Name }\end{array}$ & $\begin{array}{l}\text { Problem } \\
\text { Size }\end{array}$ & $\begin{array}{l}\text { Optimized } \\
\text { Solution }\end{array}$ & $\begin{array}{l}\text { Error of the Best } \\
\text { GA Solution (\%) }\end{array}$ & $\begin{array}{l}\text { Error the Best HBMO } \\
\text { Solution (\%) }\end{array}$ & $\begin{array}{l}\text { Error of the Best COA Error of the Best } \\
\text { Solution (\%) }\end{array}$ & $\begin{array}{l}\text { Eor } \\
\text { WCO Solution (\%) }\end{array}$ \\
\hline Esc32a & 32 & 130 & 21.69 & 54.86 & 10.769 & 3.70 \\
Esc32b & 32 & 168 & 20.75 & 50.56 & 16.66 & 1.75 \\
Esc32c & 32 & 642 & 0 & 9.7 & 0 & 0 \\
Esc32d & 32 & 200 & 0 & 29.57 & 0 & 0 \\
Esc32e & 32 & 2 & 0 & 0 & 0 & 0 \\
Esc32h & 32 & 438 & 1.79 & 22.06 & 0.9132 & 0.4235 \\
\hline
\end{tabular}

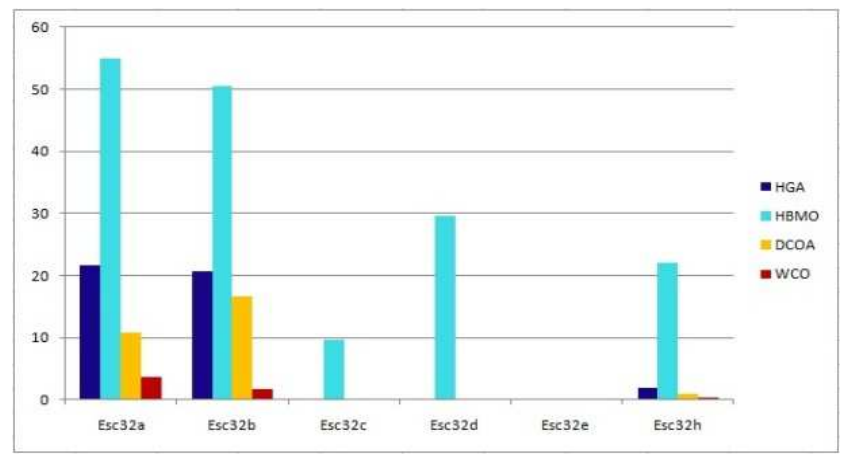

Figure 1. Comparison of Algorithms results for low-level.

WCO the problems and the weak points of other optimization algorithms (such as GA, HBMO and the other new methods like colonial completion algorithm) are not seen in this method, therefore WCO method has high capability to converge to the optimized solution faster than the other algorithms and also has this ability to find overall optimized points with higher accuracy. The ability of WCO method was examined for problems with high dimensions and the results showed that this method works very well in this issue.

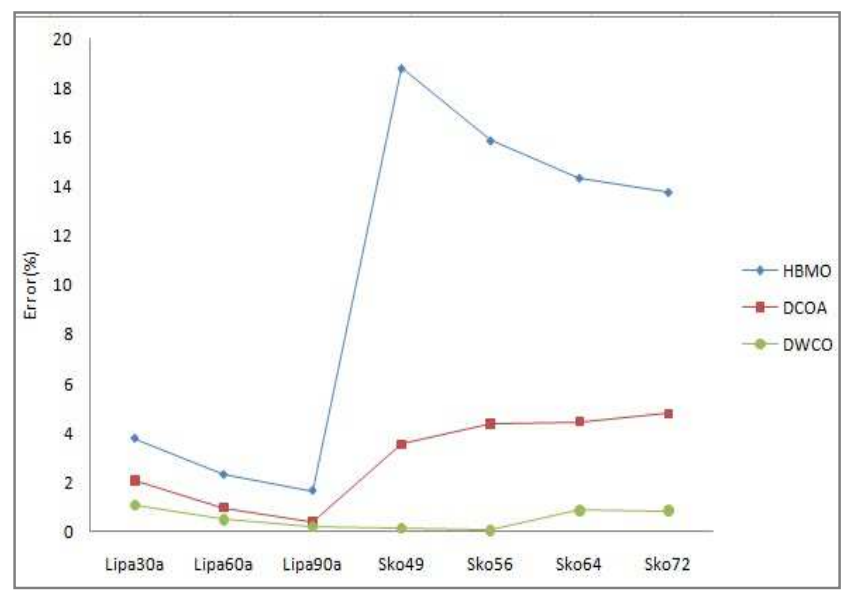

Figure 2. WCO algorithm accuracy for sko* problems.

\section{Discussion and Conclusion}

In this article the idea of WCO algorithm to solve QAP problem was studied. Results were gained for low, medium and large population. The effects of increasing optimization repeats in enhancement of algorithm`s answering precision are also investigated. The results indicate that water-cycle optimization algorithm performs better than Genetics, Cuckoo and Bee Colony algorithms, and in most comparisons, has better performance to other evolutionary algorithms in different test functions to find the best solution. Therefore, it has very fast convergence capability and the power of finding optimum points will be more precise. This algorithm generates appropriate solutions for problems with large dimensions. While the error of Genetics and Bee Colony algorithms are increased by problem with medium dimensions.

\section{References}

[1] E.Loiola, N.de Abreo, P.boaventura-nett, P.Hahn, T.Querido, "Asurvay for the Quadratic assignment problem, " Eur J Oper Res 176:657-690, 2007.

[2] RE.Burkard, T.Bonniger, "A hurestic for quadratic boolean programs with applications to quadratic assignment problems," European J, Oper.res, 13:374-86, 1983.

[3] Li.Yong, M.Panos Pardalos, and G.C.Mauricio Resende, “A Greedy Randomized Adaptive Search Procedure for the Quadratic Assignment Problem, " DIMACS Series in Discrete Mathematics and Theoretical Computer Science, May 20-21, 1993.

[4] Ghandeshtani, Mollai, Seyedkashi, and Neshati, "New Simulated Annealing Algorithm for Quadratic Assignment Problem, " The Fourth Internatinal Conference on Advanced Engineering Computing and Applications in Sciences, 2010.

[5] J.Skorin-Kapov, "Tabu search applied to the quadratic assignment problem, ” ORSA J. Comput. 1990;2:33-45. R.K. Ahuja et al. Computers \& Operations Research 27,917-934, 2000 .

[6] E.Taillard, Robust, "tabu search for the quadratic assignment problem, " Parallel Comput, 17,443-55, 1991.

[7] C.Fleurent, JA.Ferland, "Genetic hybrids for the quadratic assignment problem, " DIMACS Series in Discrete Mathematics and Theoretical Computer Science, vol. 16, Providence, RI: American Mathematical Society, pp, 173-87, 1994.

[8] T.Stutzle, M.Dorigo, "ACO algorithms for the quadratic assignment problem, " In: Corne, D., Dorigo, M., Glover, F. (Eds.), New Ideas for Optimization. McGraw-Hill, pp, 33-50, 1999. 
[9] L.Gambardella, E.Taillard, M.Dorigo, "Ant Colonies for the QAP, " Tech. Report IDSIA,4-97, IDSIA, Lugano, Switzerland, 1997.

[10] Z.W. Geem, J.-H. Kim, G.V. Loganathan, "A new heuristic optimization algorithm: harmony search," Simulation,76 (2) 60-68, 2001.

[11] A.Safari Mamaghani,and M.Reza Meybodi, “An Application of Imperialist Competitive Algorithm to Solve the Quadratic Assignment Problem," 6th international conference on internet technology and secured translation, 11-14, 2011.

[12] M.Mirzazadeh, Gh.Hasan Shirdel AND B.Masoumi, “A Honey Bee Algorithm to Solve Quadratic Assignment Problem," Journal of Optimization in Industrial Engineering (2011) 2736 .

[13] R. Rajabioun, "Cuckoo Optimization Algorithm, ” In: Applied Soft Computing journal, vol. 11, pp, 5508-5518, 2011.
[14] E.M.Loiola, N.M.Maia de Abreu, P.O.Boaventura-Netto, P.Hahn and T.Querido, "A survey for the quadratic assignment problem," European Journal of Operational Research, 176, 657-690, 2007.

[15] T. C. Koopmans and M. J. Beckmann, "Assignment problems and the location of economic activities," Econometrica, 25, 53-76, 1957.

[16] M.Hosseini, M.Sadri, "A new evolutionary algorithm based on the water cycle in nature," 4th Conference on Electrical and Electronics Engineering, University GONABAD,7-9 August 2001.

[17] RE.Burkard, SE.Karisch, F.Rendl. "QAPLIB - A quadratic assignment program library,” J.Global Optim.;10:391$403,1997$. 